Forum $2017 \cdot 32: 366$

DOI 10.1007/s12312-017-0304-0

Online publiziert: 28. Juli 2017

○) Springer Medizin Verlag GmbH 2017

\title{
VTESARO"
}

TESARO ist ein in Europa neues biopharmazeutisches Unternehmen mit amerikanischen Wurzeln in Waltham/ Massachusetts, das ab jetzt auch in Europa moderne Therapieoptionen für onkologische Patienten anbietet. Der internationale Firmensitz befindet sich in Zug in der Schweiz, die deutsche Niederlassung TESARO Bio Germany GmbH ist in München. Von hier aus werden auch die Nachbarländer Österreich und Schweiz betreut.

Der Name des Unternehmens steht für seine Philosophie, der Suche nach verborgenen Schätzen. „TESARO“ stammt von dem galizischen Word „tesouro“ ab, was so viel wie „Schatz, Kostbarkeit“ bedeutet. Das Unternehmen möchte Werte schaffen - für Patienten, Therapeuten und die Mitarbeiter, die für das Unternehmen oder einen seiner Partner arbeiten. Diese Vision von TESARO zeigt sich in einer starken Pipeline, umfangreichen Ressourcen und einer soliden finanziellen Entwicklung.

Ein wesentlicher Bestandteil des Erfolgs von TESARO ist der Gedanke der Partnerschaft. TESARO sieht sich als zuverlässiger Partner für onkologisch tätige Fachkreise und für Patienten mit Krebserkrankungen. Wie die deutsche Geschäftsführerin Birgit Schunck erklärt: „Uns ist es wichtig, unsere Pipeline durch Partnerschaften voranzubringen und zu stärken, um sicherzustellen, dass wir den Bedürfnissen von Therapeuten und Patienten

\section{Tesaro Bio GmbH - ein neues Sektion C Mitglied stellt sich vor}

heute und auch in Zukunft nachkommen können. Dies drückt sich auch in unserem Motto: „Together we can make a difference" aus.

TESARO entwickelt, kauft und vertreibt onkologische Therapeutika und Supportivprodukte, die das Leben von Tumorpatienten entscheidend beeinflussen können. Hierbei spielt die Lebensqualität eine wichtige Rolle. Soeben wurde mit dem oralen Antiemetikum Varuby ${ }^{\otimes}$ (Rolapitant) das erste Präparat in Deutschland eingeführt. Rolapitant ist ein P/Neurokinin-1(NK-1)-Rezeptorantagonist, der als Teil einer Kombinationstherapie für die Prävention von verzögerter Übelkeit und Erbrechen bei hoch und moderat emetogener Chemotherapie bei erwachsenen Tumorpatienten indiziert ist. Bekanntlicherweise ist eine wirksame Antiemese entscheidend für die Verträglichkeit und Akzeptanz einer emetogenen Chemotherapie.

In der Pipeline befinden sich unter anderem, Niraparib, ein in den USA vor kurzem bereits zugelassener, oraler Poly(ADP-Ribose)-Polymerase (PARP)Inhibitor, der derzeit beim Ovarial- und Mammakarzinom untersucht wird. $\mathrm{Zu}-$ dem wurde bereits die FDA-Zulassung von intravenösem Rolapitant beantragt. TESARO verfügt auch über verschiedene immunonkologische Substanzen wie z. B. den Programmed Cell Death 1 (PD1)-Antikörper TSR-042, der Potenzial in ver-

\section{Zahlen und Fakten TESARO}

TESARO Inc.

Gegründet 2010 in Waltham/MA

CEO: Leon (Lonnie) O. Moulder, Jr

TESARO Bio Germany GmbH

Gegründet 2016

Geschäftsführerin Deutschland/Österreich/

Schweiz: Birgit Schunck

Präparate: Varubi ${ }^{\circledast}$ (FDA 2015), Varuby ${ }^{\circledast}$ (EMA

2017), Zejula ${ }^{\circledast}$ (FDA 2017)

schiedenen onkologischen Indikationen besitzt.

TESARO fördert durch die Initiative „GROW“(Global Research Opportunities for Wellness) sogenannte „drug hunting“ Partnerschaften. GROW verfolgt einen strategischen, auf Beziehungen aufbauenden Ansatz bei der Identifizierung und Förderung präklinischer Forschungsmöglichkeiten für die Schaffung neuer therapeutischer Konzepte und onkologischer Forschungsgebiete, die für die Verbesserung des Lebens von Tumorpatienten vielversprechend scheinen.

\section{Korrespondenzadresse}

TESARO Bio GERMANY GmbH

Leopoldstraße 23

80802 München, Deutschland

+49 (089) 24442-3074

kontakt-Deutschland@tesarobio.com 